\title{
Effectiveness of medical interventions implemented during ototoxicity monitoring to prevent further deterioration of hearing thresholds
}

Hlupheka M. Maluleka (MLLHLU001@myuct.ac.za)

Silva Kuschke (silvakuschke@gmail.com) ORCID: orcid.org/0000-0002-1046-7435

Lebogang Ramma (Lebogang.Ramma@uct.ac.za)

Smangele Mashele, Matsie M. Ntatamala, Tracy A. Frosler, Ezethu B. Potelwa, Dylan G. Walker

Supervisors: Ms Silva Kuschke; A/Prof Lebogang Ramma

Health \& Rehabilitation Sciences, University of Cape Town

\section{ABSTRACT}

Background: MDR-TB patients are often treated with aminoglycosides which may lead to irreversible ototoxic hearing impairment. Early identification of changes in the hearing thresholds of patient is critical in facilitating treatment modifications that can minimise communication impairment.

Design: A retrospective review of medical records of all patients who underwent ototoxicity monitoring from 2012-2014 was conducted. Patients treated with aminoglycosides and with normal hearing thresholds at baseline were included.

Results: 26 of 509 patient records met inclusion criteria. There were 9 males and 17 females. The mean age was $36.81 \pm 11.48$. Half of the participants were on treatment for $\leq 160$ days, whereas the rest were on treatment for $\geq 161$ days. The greatest deterioration in hearing thresholds (post-treatment initiation) occurred in the high frequencies $(4-8 \mathrm{kHz})$. Participants on treatment for $>160$ days had the greatest deterioration in hearing thresholds. Modifying the frequency of drug administration led to a lesser degree of deterioration of hearing thresholds.

Conclusion: Early detection of deterioration in hearing thresholds can potentially minimise ototoxicity in patients who are being treated with aminoglycosides. More research is needed regarding the effectiveness of these strategies.

Keywords: Ototoxicity, multidrug resistant tuberculosis (MDR-TB), treatment modifications, aminoglycosides, hearing thresholds 


\section{Introduction}

Although Tuberculosis (TB) can be prevented and cured, it continues to cause high numbers of deaths globally. The World Health Organisation (WHO) ranks TB as the second leading cause of mortality after human immunodeficiency virus and acquired immune deficiency syndrome (HIV/AIDS) (WHO, 2014:1). South Africa is in crisis due to the TB epidemic with prevalence rates currently estimated to be 715 per 100000 population, thus qualifying South Africa as one of the countries with the highest TB prevalence in the world (WHO, 2014).

Emergence of strains of TB that are resistant to anti-tuberculosis drugs, i.e. drug resistant TB (DR-TB), is also a source of concern for the South African national TB programme (SANTP) (Pooran et al., 2013:10). WHO (2014:57) estimates the incidence of multidrug resistant TB (MDR-TB) in South Africa to be $1.8 \%$ amongst new TB cases and $6.7 \%$ amongst re-treatment cases. South Africa is also one of the countries with a high number of patients diagnosed with either multidrug-resistant TB (MDR-TB) or rifampicin-resistant TB (RR-TB) globally, with at least 26,023 cases of MDR-TB or DR-TB diagnosed during 2013 (WHO, 2014:65).

Concern regarding the emergence of DR-TB is due to the fact that it requires more toxic treatment, which is associated with poor outcomes (Pooran et al., 2013:1) and other secondary effects such as hearing loss, which may or may not be reversible (Khoza-Shangase, Mupawose \& Mlangeni, 2009:392). Aminoglycosides are well known to be the most toxic drugs used for treatment of MDR-TB (Xie, Talaska, \& Schacht, 2011:29). One of the known side-effects of aminoglycoside is permanent, bilateral sensorineural hearing loss which usually starts in the high frequency region (Duggal \& Sarkar, 2007). Incidence of aminoglycoside-induced hearing loss following initiation of treatment has been reported to range between 1857\% (Duggal \& Sakar, 2007; Harris et al, 2012:364)

Although there are other less toxic alternative drugs, use of aminoglycosides is preferred because they are inexpensive and a cost-effective choice for developing countries (Xie, Talaska, \& Schacht, 2011:28). In accordance with the WHO guidelines, kanamycin and amikacin are aminoglycosides used in South Africa for MDR-TB treatment (WHO, 2006:35; Human, et al., 2010:752). 
Hearing loss following treatment with aminoglycoside drugs can have devastating effects on patients. In children, any hearing loss regardless of the severity can impede educational performance, psychosocial behaviour and speech and language development (Elfenbein, Hardin-Jones, \& Davis, 1994; Whitehorn et al., 2014:288). In adults, hearing loss can impair the exchange of information, which includes communication input (hearing) and output (speech and language). This could significantly impact everyday life, causing loneliness, isolation, dependence, and frustration (Ciorba et al., 2012:162). Thus, understanding the extent of aminoglycoside-induced hearing loss as an outcome of MDR-TB patients and finding ways to minimise it is crucial as well as in the best interest of both the patient and health professionals.

There are individual predispositions and treatment factors that are known to increase the risk of developing aminoglycoside induced hearing loss. Individual predisposition factors include genetics (mutations in the 12S rRNA gene), age, HIV positive status, hypovolaemia (a decreased volume of blood circulation in the body), bacteraemia (the presence of bacteria in the blood), liver dysfunction and noise exposure (Fischel-Ghodsian et al., 1997:177; Bates, Beaumont, \& Baylis, 2002:448; Moore, Smith \& Lietman, 1984:27; Harris et al., 2012:365). Treatment factors include length of treatment (cumulative dose) and repeated courses of treatment. According to Bardien et al. (2009:440), treatment lasting longer than seven days puts patients at risk for ototoxicity. This is also supported by De Jagger and Van Altena (2002:642) who investigated the effects of long-term use of aminoglycosides and found significant hearing loss in patients treated with aminoglycosides, specifically amikacin, kanamycin and/or streptomycin.

There is sufficient evidence that shows a high degree of variability regarding the relationship between medication/treatment administration parameters such as dose, treatment duration, and blood serum concentration and the likelihood of developing ototoxic effects (Whitehorn et al., 2014:290; Schacht, Talaska, \& Rybak, 2012:1839; Selimoglu, 2007:122; Rizzi \& Hirose, 2007:353). It is therefore difficult for the attending medical doctor to rely solely on medication/treatment administration factors to predict the risk of ototoxicity (American Speech-Language-Hearing Association $[A S H A], 1994)$. Therefore, prospective assessment of hearing status for 
all individuals on treatment that includes ototoxic medications remains the only reliable method for early detection of change in hearing thresholds prior to the presentation of symptomatic hearing loss (ASHA, 1994; Fausti et al., 1994:232; Konrad-Martin et al., 2005:18).

Hearing thresholds of patients are monitored so that any changes in their hearing thresholds presumably attributed to medication or treatment regimen could be detected early to give the attending medical doctor a chance, if feasible, to alter the treatment regimen of the patient. In cases where a patient acquires a disabling hearing impairment - as it is not always possible to prevent hearing impairment via ototoxicity monitoring - appropriate audiologic intervention could be provided timeously to patients and their families (American Academy of Audiology [AAA], 2009:3). Ototoxicity monitoring is therefore grounded on the twin principles of early identification and early intervention (AAA, 2009:3).

If the hearing thresholds of a patient are found to deteriorate during ototoxicity monitoring following treatment initiation, a doctor has several options in terms of interventions which may be implemented to minimise further deterioration of the patient's hearing status (Konrad-Martin et al., 2005:18). These medical interventions may include reducing the dose of medication/drug administered, changing the frequency of drug administered or discontinuing treatment with the current drugs, switching to less ototoxic drugs, or continuing treatment and preparing the patient and family to cope with the hearing loss (Konrad-Martin et al., 2005:18). This study therefore aimed to determine the types and effectiveness of medical interventions implemented in response to a significant deterioration in hearing thresholds of patients during MDR-TB treatment at Brooklyn Chest Hospital $(\mathrm{BCH})$.

\section{Method}

\section{Research design}

A retrospective medical folder review research design was followed. This design is non-experimental, and the researcher uses existing data to examine the association between a disease, medical event or outcomes and the exposure to it (Song \& Chung, 2010:2236). Observational studies are useful in answering questions relating to prevalence, incidence, cause, prognosis, or effect of disease and treatment 
(Mann, 2003:54). However, this design is subject to confounding variables/factors, henceforth results should be interpreted with caution (Song \& Chung, 2010:2234).

\section{Participants}

This study did not involve direct contact with participants but rather used medical folders of patients with MDR-TB who were previously monitored for ototoxicity at Brooklyn Chest Hospital (BCH).

\section{Sample and sampling method}

The study sample consisted of medical folders of patients who were diagnosed with MDR-TB and monitored for ototoxicity from 2012-2014. Convenience sampling was used in order to ensure that the eligibility criteria were met. Convenience sampling is a non-probability sampling technique that is frequently used in quantitative studies (Suen, Huang \& Lee, 2014). The method was chosen because it is quick and easy to implement and thus the researchers are able to analyse the data timeously (Suen, Huang \& Lee, 2014). A drawback is that the results can be biased and therefore cannot be generalized to a population (Suen, Huang \& Lee, 2014).

\section{Inclusion \& exclusion criteria}

Only records of MDR-TB in-patients who had at least three audiograms; 1) baseline audiogram showing normal hearing and normal tympanograms , 2) monitoring audiogram showing the first change ${ }^{1}$ in hearing thresholds (threshold shift), and 3) the last audiogram in the patients' records were included for review. Patients who were repeating a course of treatment were excluded from the study.

\section{Data collection}

\section{Materials}

A data abstraction form that was developed specifically for this study (see Appendix A), was used to extract data from the medical records. The abstraction form was designed to extract the following information; demographic information of patients, at least 3 audiograms with dates (baseline audiogram, first change monitoring audiogram and last monitoring audiogram after intervention); tympanometry results;

\footnotetext{
${ }^{1}$ First change in hearing thresholds is defined as an audiogram showing the first change in the patient's hearing thresholds (i.e. first hearing threshold shift) following MDR-TB treatment initiation
} 
and type of drug and intervention received. The use of a standard abstraction form ensured a measure of consistency among the researchers, and reduced the chances of transcription error and input.

\section{Pilot study}

A pilot study was done to check the suitability of the data abstraction tool and feasibility of the study prior to conducting a larger scale study to identify any possible modifications (Leon, Davis \& Kraemer, 2011: 629). In this study, data was collected using a paper based abstraction sheet and then the data was transferred to an electronic spreadsheet from which analysis was done. Two hundred folders were reviewed and it was discovered that using a paper based abstraction form was not feasible as it was time consuming and uneconomical. The pilot study resulted in data being directly captured into an electronic version as a modification.

\section{Procedures}

Ethical clearance to conduct the study was first requested from the University of Cape Town Faculty of Health Sciences Human Research Ethics Committee (HREC: 878/2014). After ethical clearance was granted, permission from hospital management and then the audiologists was requested for full access to the database. Data collection for this study proceeded as follows:

Medical folders of patients who were monitored for ototoxicity during the specified period [2012-2014] were obtained from the Audiology Department at BCH with the help of the audiologists. The records were sorted according to the study inclusion and exclusion criteria. Information of interest, demographic information, audiometric information, type of drug, type of medical intervention etc. was recorded on the electronic version of the data abstraction form using laptops. Data from different spreadsheets was merged into one spreadsheet and participant numbers were assigned for the patients. ASHA, 1994 criteria was used to define a change in hearing thresholds.

\section{Classification of hearing loss and calculation of Pure Tone Average (PTA)}

Pure-tone average (PTA) is the average in decibels $(\mathrm{dB})$ of hearing thresholds at a certain range of frequencies. An average at $0.5 \mathrm{kHz}, 1 \mathrm{kHz}$ and $2 \mathrm{kHz}$ was 
calculated to determine the speech frequency PTAs (Brown et al., 2010:1222). It was decided in this study to calculate high frequency PTA using a three-frequency average based on thresholds at $4 \mathrm{kHz}, 6 \mathrm{kHz}$ and $8 \mathrm{kHz}$. These frequencies were chosen because high frequencies are most likely to be affected by ototoxicity (Schacht, Talaska, \& Rybak, 2012:1839). Thereafter, the change in low and high frequency PTA before and after medical intervention was calculated and presented graphically.

The effect of cumulative dose of high frequencies was analysed and length of treatment was determined by the number of days between the date of the baseline audiogram and the last audiogram.

\section{Data Management}

The data was stored on the VULA group site which is only accessible to the researchers and supervisors.

\section{Data Analysis}

Data was analysed using descriptive and inferential statistics. Descriptive statistics were done using excel functions while inferential statistics were done using the IBM SPSS programme. T-tests were used to compare if the variables observed were significantly different from each other (Durrheim, 2002:143). The repeated measure factorial ANOVA was used as there were multiple factors (independent variables) observed from the same participants (Durrheim, 2002:290). The factors included the type of medical intervention and the change in hearing. A multivariate ANOVA was done to compare the overall change in hearing loss of the participants who received the different interventions. In the conditions of the factorial ANOVA, the probability of the significance values decreases when more than two groups are being compared and in these conditions there are chances of making type II error (failure to reject a false null hypothesis or "false positive") (Durrheim, 2002:134).

\section{Ethical considerations}

The study was conducted in accordance with the World Medical Association Declaration of Helsinki (2013) and the following ethical principles were adhered to: Autonomy, beneficence, non-maleficence and justice. Measures were also put in place (e.g. storing of data for this study in a locked cabinet) to ensure that patient information was treated with the highest degree of confidentiality (Bramley \& Matiti, 2014:2793),. 


\section{Results}

509 patient folders were reviewed and only 26 met the selection criteria (see Figure 1).

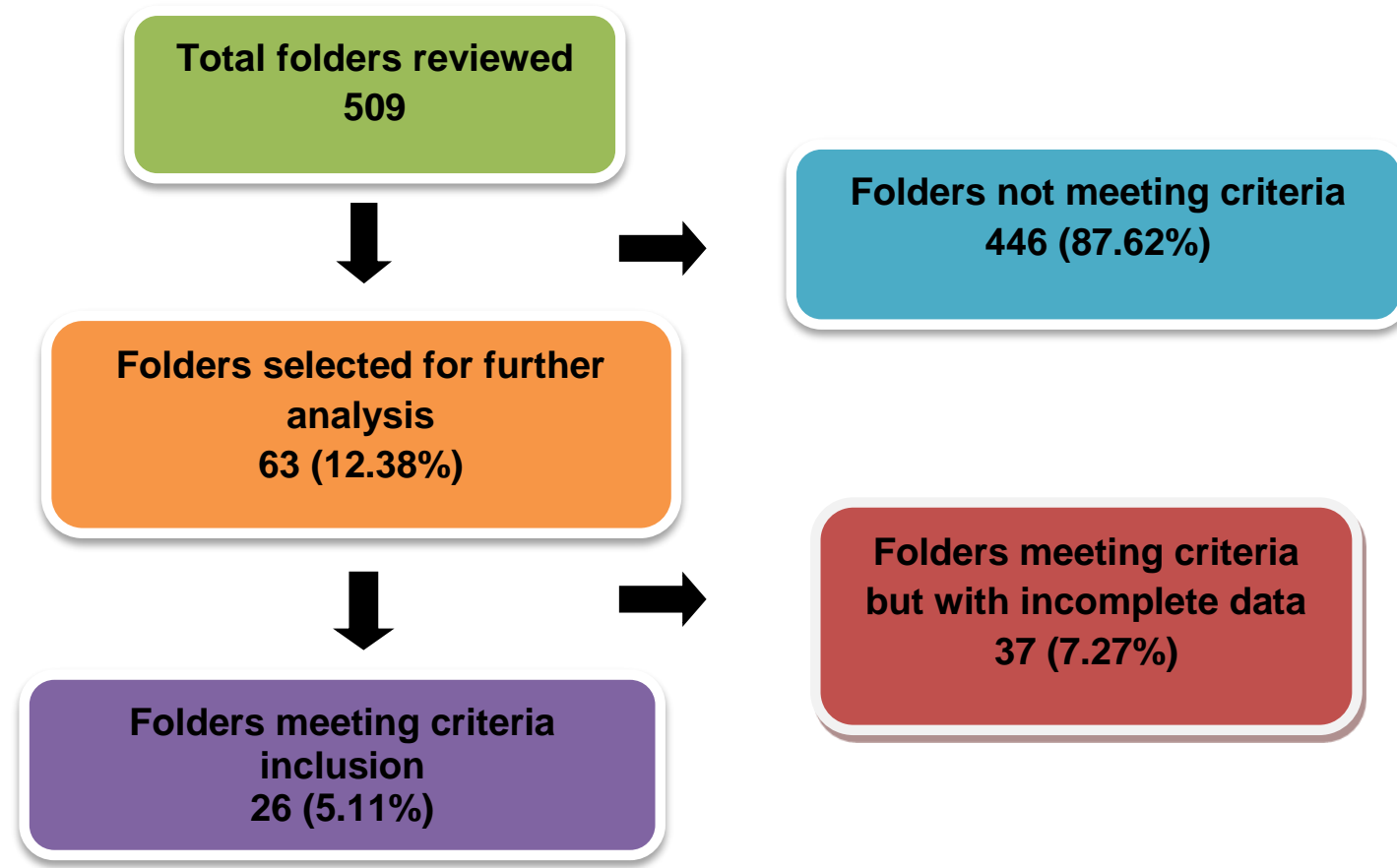

\section{Figure 1: Selection of folders for review}

The participant sample variables and variables relating to duration of treatment are summarised in Table 1. There were more females than males and the mean age was 36.81 ( $S D=11.48)$. Half of the participants were on treatment for less than 6 months and the remaining half were on treatment for more than 6 months. The mean duration of treatment was 301.92 days $(S D=283.88)$.

All participants showed a change in hearing thresholds after initiation of treatment and the magnitude of the change was greater in the high frequencies than the low frequencies. The deterioration of hearing thresholds after the intervention was less than the change before intervention (see Figure 2 in the next page). 
Table 1: Description of the different factors and participants, $\mathbf{n = 2 6}$.

\begin{tabular}{|l|l|}
\hline Factors & $\mathbf{n}(\%)$ \\
\hline Gender & \\
\hline Male & $9(34.62)$ \\
\hline Female & $16(61.54)$ \\
\hline Unknown & $1(3.85)$ \\
\hline Age & \\
\hline Mean age (SD) & $36.81(11.48)$ \\
\hline Range & $42[62-20]$ \\
\hline $\begin{array}{l}\text { Duration between baseline } \\
\text { and last audiogram (days) }\end{array}$ & \\
\hline 1 - 160 & $13(50)$ \\
\hline 161+ & $13(50)$ \\
\hline Duration on treatment (days) & \\
\hline Mean (SD) & $301.92(283.88)$ \\
\hline Range & $976[1036-60]$ \\
\hline All patients were treated with kanamycin \\
\hline
\end{tabular}

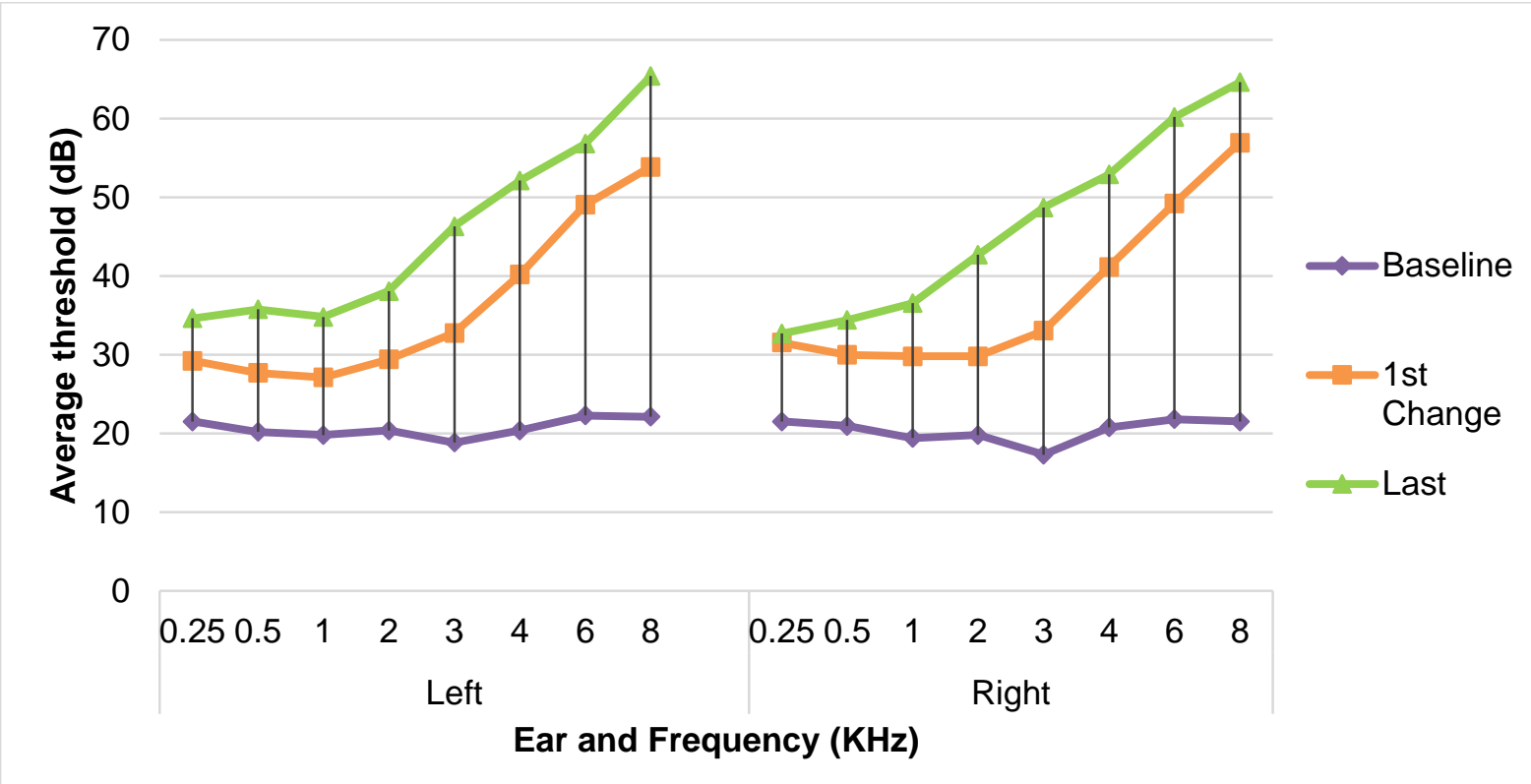

Figure 2: Threshold average at baseline, 1st change and last audiograms. 
There was a difference in the amount of change in hearing thresholds before medical intervention and after medical intervention in both the low and high frequency PTA. The difference was more pronounced in the high frequencies (Figure 3 ). However, the initial change in the high frequency PTA was not significantly different to the second change, for the left ear $t(25)=1.47, p=.154$ and for the right ear $t(25)=$ $1.81, p=.082$.

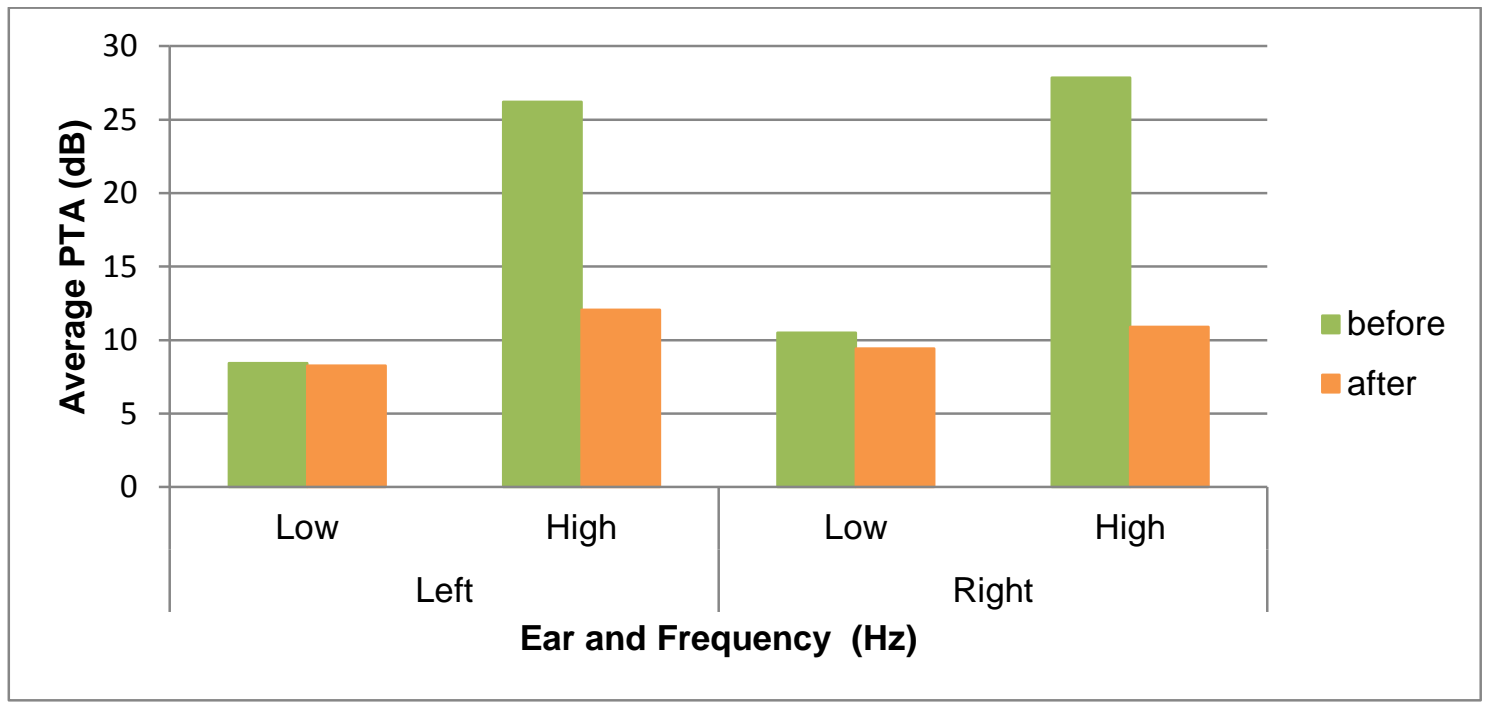

Figure 3: Change in PTA before and after a medical intervention.

The change in high frequency PTA as a function of duration of treatment is presented in Figure 4, based on the dates of the baseline and last audiograms. From this figure it is evident that participants who were on treatment for 161 days or more showed greater overall change in high frequency PTA than those who were on treatment for 160 days or less in both the left and right ear. Independent group t-tests were performed to examine the overall change in high frequency PTA in the left and right ears. The result was significant for the left ear, $t(24)=-3.02, p=.006$, but was not for the right ear, $t(24)=-1.83, p=.079$. 


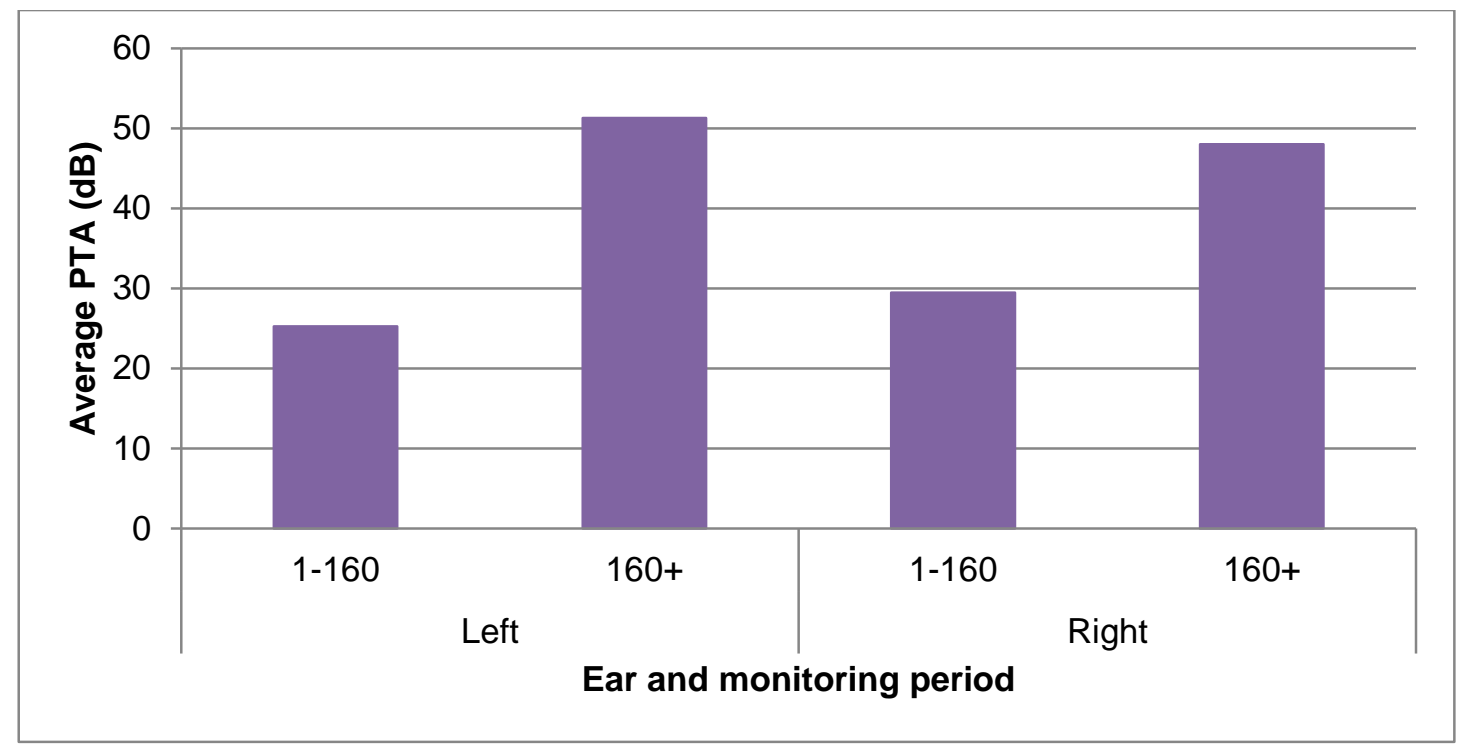

Figure 4: Overall change in high frequency pure tone average for short and long audiological monitoring period.

There were three types of medical interventions that were implemented when hearing loss was first detected, namely, 'change frequency of the drug administered', 'stop treatment' and 'continue treatment as is' (refer to Figure 5). The most common intervention was changing frequency of drug administered, followed by continuing treatment as is and then stopping treatment.

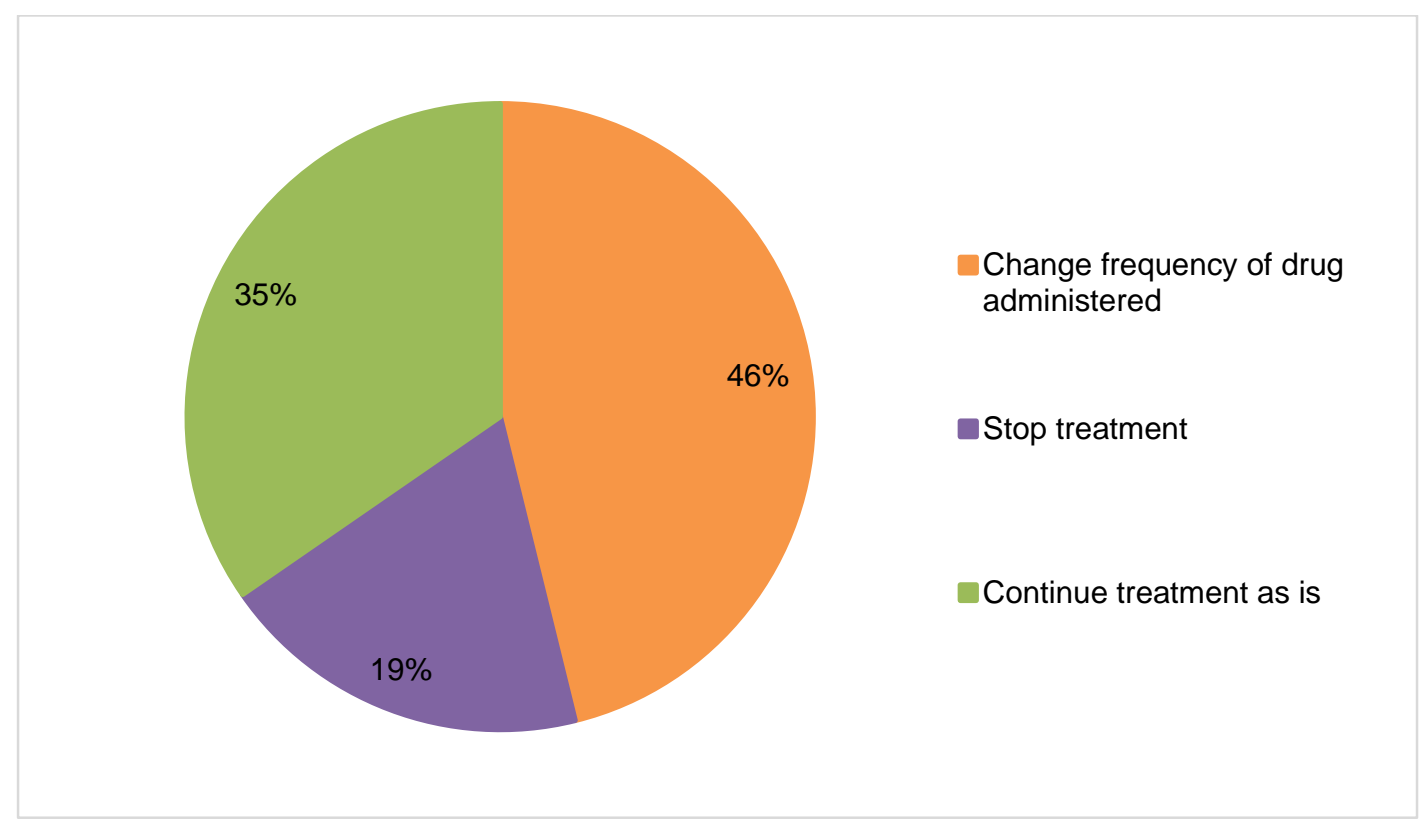

Figure 5: Types of medical interventions. 
Figure 6 shows the change in the hearing thresholds of the patients before and after medical intervention. 'Change frequency of the drug administered', 'stop treatment' and 'continue treatment as is' are represented by strategy A, B and C, respectively. In the left ear, participants who received strategy $B$ had the greatest change before intervention; however, they had the least change after intervention. In the right ear, participants who received strategy $\mathrm{A}$ had the second greatest change before intervention; however, they had the least change after intervention. On the other hand, participants who received strategy A showed the least amount of overall change in hearing thresholds from baseline bilaterally.

The high frequency PTAs for the different interventions at the different change times were then examined with a $3 \times 2$ (Intervention [strategy A, strategy $B$, strategy $\mathrm{C}] \times$ Change [before, after]) repeated measures ANOVA. This ANOVA revealed that none of the interventions differ significantly, before and after interventions in both the left and the right ear, $F(2,23)=0.001, p=.999$ in the left ear, and for the right ear $F$ $(2,23)=0.082, p=.922$. Furthermore, the overall change in the high frequency PTAs did not differ significantly for the different intervention in neither the left and right ears, $F(2,23)=0.358, p=.703$ in the left ear and for the right ear $F(2,23)=0.305, p=$ .740 .

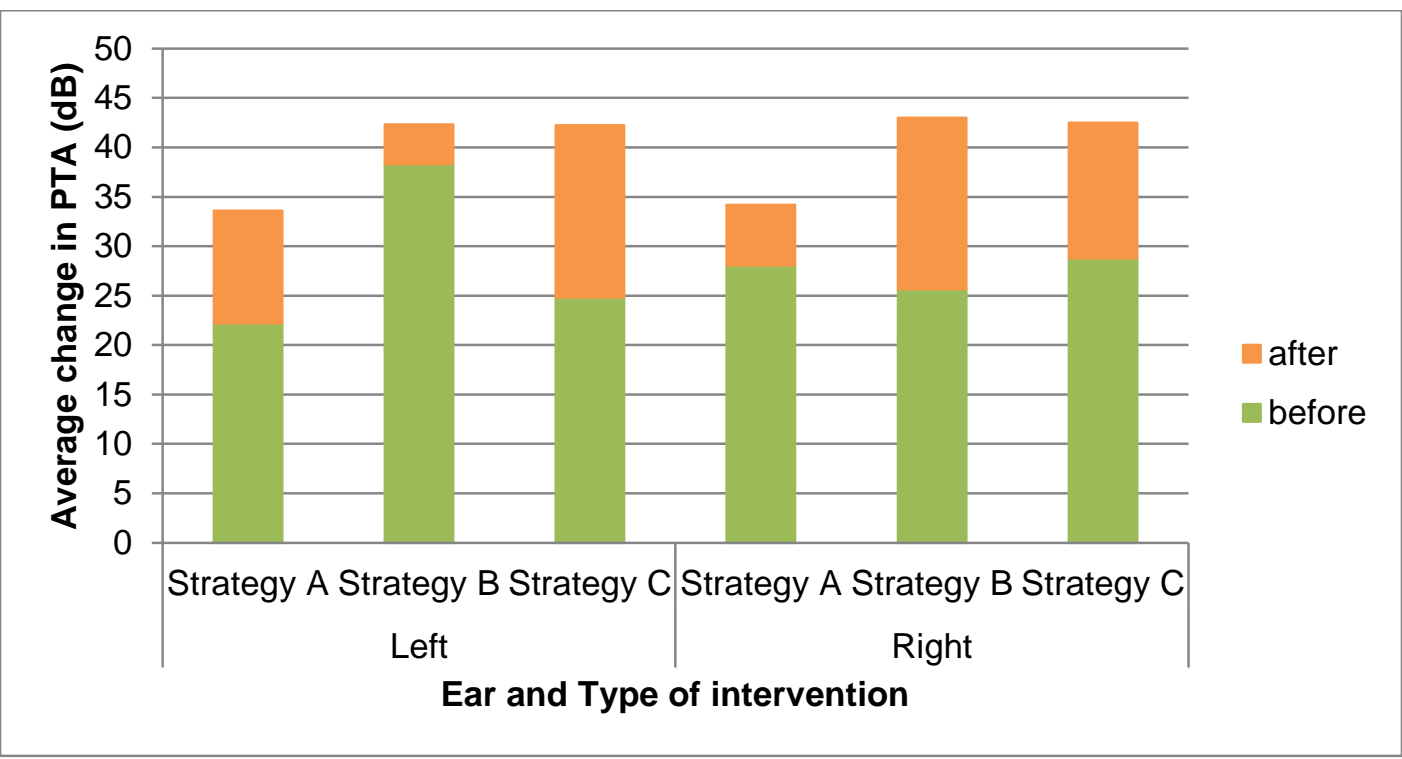

Figure 6: Change in high frequency pure tone average before and after the three types of intervention. 


\section{Discussion}

This study showed that aminoglycoside-induced hearing impairment affects mainly the high frequencies, and the degree of deterioration of hearing thresholds increases as the duration of treatment increases. The study also showed that the most common medical intervention at $\mathrm{BCH}$ was modifying the frequency of drug administration, which also seemed to be the most effective strategy.

Patients with MDR-TB who were treated with ototoxic aminoglycosides showed deterioration in their hearing thresholds. The deterioration of hearing thresholds was more rapid in the high frequencies. The findings of this study are consistent with those of Schacht, Talaska, \& Rybak (2012:1839), who suggest that aminoglycoside induced hearing loss is bilateral and begins at high frequencies and with longer treatment, eventually extends to the lower frequencies. The fact that hearing loss occurs more rapidly in the high frequencies may be due to the fact that the outer cochlear hair cells which are located at the basal region of the cochlear (which processes high frequencies information) are damaged first (Schacht, Talaska, \& Rybak, 2012:1840).This could potentially suggest that tests which are more sensitive to detect changes in the high frequency hearing thresholds are crucial for monitoring the hearing status of patients at risk for ototoxicity (ASHA, 1994).

This study also showed that patients who were on treatment for longer had a greater shift in hearing thresholds than those who were on treatment for a shorter period. Cumulative dose, which is dependent on the length of treatment, is known to be one of the significant risk factors for developing ototoxicity (Bardien et al, 2009:440).

Of the five available medical intervention options outlined in the literature review (Konrad-Martin et al., 2005:18), only three were implemented when hearing loss was first detected. 'Change frequency administered' was the most common medical intervention implemented, followed by the 'continue treatment as usual' and 'stopping treatment' was the least common intervention implemented.

Modifying the frequency of drug administration schedule led to a slightly lesser degree of hearing loss when compared to the other two strategies. However, since there was no statistically significant difference between the overall shifts in hearing 
thresholds resulting from implementation of different medical intervention strategies, it is not possible to confidently say which medical intervention was the most effective. This lack of statistical significance could be due to patient factors that increased the risk of developing ototoxicity (Fischel-Ghodsian et al., 1997: 177; Bates, Beaumont, \& Baylis 2002:448; Moore, Smith \& Lietman, 1984:27; Harris et al., 2012:363), which were not controlled for in this study, or possibly because the study sample was small and therefore did not have sufficient statistical power to show a significant difference. Another reason could be the fact that less sensitive audiometric tests were used to monitor patient status (i.e. no ultrahigh frequency testing was done, $0.25 \mathrm{kHz}-8 \mathrm{kHz}$ was used) which means that by the time the patients' hearing thresholds started to change it was already too late to do anything about it.

The findings of this study must be interpreted with caution given its methodological limitation; small sample size and retrospective review of data which did not give the researchers control over the quality of data collected and used in this study. Furthermore, although it was shown in this study that one of the medical intervention strategies may have led to slightly better outcomes when compared to others, these effects cannot be wholly attributed to the medical intervention stated because when adverse treatment effects are discovered, doctors try other several interventions which may also result in the decreased progression of hearing loss. That is, the intervention implemented when a change in hearing was first observed is not necessarily the only intervention which the patient received. However, in spite of its limitations, this small study is one of the few studies conducted in South Africa to document outcome of medical interventions implemented with respect to preventing/reducing the deterioration of patient hearing thresholds during treatment with ototoxic medications.

\section{Implications and recommendations for future studies}

Tests such as ultra-high frequency audiometry are crucial for early detection of and intervention for ototoxicity because they are sensitive to detection of hearing loss in the high frequency region before it gets to the standard frequency range (i.e. 0.25 $\mathrm{kHz}-8 \mathrm{kHz}$ ). Therefore future studies must investigate whether using tests that are sensitive to high frequency hearing loss are useful with regards to giving early warning before the hearing loss starts affecting the low frequency region. 


\section{Conclusion}

This study explored the effectiveness of different medical interventions implemented during ototoxicity to prevent/reduce deterioration of hearing thresholds of patients during ototoxicity monitoring. These interventions have the potential to slow down the progression of aminoglycoside induced hearing loss and therefore can reduce future communication impairment. However, for these strategies to be effective, early identification of changes in hearing thresholds of patients through tests that are more sensitive to high frequencies are critical during ototoxicity monitoring. Good communication and working relationships between doctors and audiologists is also critical so that these interventions can be implemented on time when indicated.

\section{References}

American Academy of Audiology. 2009. Position statement and clinical practice guidelines: ototoxicity monitoring [homepage on the Internet]. Available from: http://www.audiology.org/publications-resources/document-library/ototoxicitymonitoring [2015, November 18].

American Speech-Language-Hearing Association. 1994. Guidelines for the audiologic management of individuals receiving cochleotoxic drug therapy [Guidelines]. Asha, 36 (Suppl. 12), 11-19. Available: http://www.asha.org/policy/GL199400003.htm\#AP1 [2015, November 18].

Bardien, S., Jong, G.D., Schaaf, H. S., Harris, T., Fagan, J., \& Petersen, L. 2009. Aminoglycoside-induced hearing loss: South Africans at risk. South African Medical Journal, 99(6): 440-441.

Bates, D. E., Beaumont, S. J., \& Baylis, B. W. 2002. Ototoxicity induced by gentamicin and furosemide. Annals of Pharmacotherapy, 36(3):446-451.

Brown, R. F., Hullar, T. E., Cadieux, J. H., \& Chole, R. A. 2010. Residual hearing preservation after paediatric cochlear implantation. Otology \& Neurotology: Official Publication of the American Otological Society, American Neurotology Society [and] European Academy of Otology and Neurotology, 31(8): 12211226. http://doi.org/10.1097/MAO.0b013e3181f0c649 
Ciorba, A., Bianchini, C., Pelucchi, S., \& Pastore, A. 2012. The impact of hearing loss on the quality of life of elderly adults. Clinical Intervention Aging, 7, 159-163. DOI: $10.2147 /$ CIA.S26059

Chang, K.W., \& Chinosornvatana, N. 2010. Practical grading system for evaluating cisplatin ototoxicity in children. Journal of Clinical Oncology, 28(10): 1788-1795.

De Jager, P., \& Van Altena, R. 2002. Hearing loss and nephrotoxicity in long-term aminoglycoside treatment in patients with tuberculosis. The International Journal of Tuberculosis and Lung Disease, 6(7): 622-627.

Duggal, P., \& Sarkar, M. 2007. Audiologic monitoring of multi-drug resistant tuberculosis patients on aminoglycoside treatment with long term follow-up. BMC Ear, Nose and Throat Disorders, 7(1):5. Available: http://www.biomedcentral.com/1472-6815/7/5 [2015, July 22].

Durrheim, K. L. 2002. Factorial analysis of variance. In Numbers, hypotheses and conclusions. C.G. Tredoux \& K.L. Durrheim ,Eds. Cape Town: Juta Publishers. 289-307.

Elfenbein, J. L., Hardin-Jones, M. A., \& Davis, J. M. 1994. Oral communication skills of children who are hard of hearing. Journal of Speech, Language, and Hearing Research, 37(1): 216.Available: EBSCOHost Communication \& Mass Media Complete [2015, July 22].

Fausti, S. A., Larson, V. D., Noffsinger, D., Wilson, R. H., Phillips, D. S., \& Fowler, C. G. 1994. High-frequency audiometric monitoring strategies for early detection of ototoxicity. Ear and Hearing, 15(3): 232-239.

Fischel-Ghodsian, N., Prezant, T. R., Chaltraw, W. E., Wendt, K. A., Nelson, R. A., Arnos, K. S., \& Falk, R. E. 1997. Mitochondrial gene mutation is a significant predisposing factor in aminoglycoside ototoxicity. American Journal of Otolaryngology, 18(3):173-178.

Frymark, T., Leech, H., Mullen, R., Schooling, T., Venediktov, R., \& Wang, B. 2010. Evidence-Based Systematic Review: Drug-Induced Hearing Loss-Amikacin. ASHA's National Center for Evidence-Based Practice in Communication Disorders. 
Harris, T., Bardien, S., Schaaf, H. S., Petersen, L., De Jong, G., \& Fagan, J.J. 2012. Aminoglycoside: induced hearing loss in HIV-positive and HIV-negative multidrug-resistant tuberculosis patients. South African Medical Journal, 102(6):363-365 .

Human, H., Hagen, C. M., Jong, G. D., Harris, T., Lombard, D., Christiansen, M., \& Bardien, S. 2010. Investigation of mitochondrial sequence variants associated with aminoglycoside-induced ototoxicity in South African TB patients on aminoglycosides. Biochemical and Biophysical Research Communications, 393(4):751-756.

Khoza-Shangase, K., Mupawose, A., \& Mlangeni, N.P. 2009. Ototoxic effects of tuberculosis treatments: How aware are patients? African Journal of Pharmacy and Pharmacology, 3: 391-399.

Konrad-Martin, D., Gordon, J., Reavis, K., Wilmington, D., Helt, W., \& Fausti, S. 2005. Audiological monitoring of patients receiving ototoxic drugs. Perspectives on Hearing and Hearing Disorders: Research and Diagnostics. 1: 17-22. DOI:10.1044/hhd9.1.17.

Leon, A. C., Davis, L. L., \& Kraemer, H. C. 2011. The role and interpretation of pilot studies in clinical research. Journal of Psychiatric Research, 45(5): 626-629.

Mann, C. J. 2003. Observational research methods. Research design II: cohort, cross sectional, and case-control studies. Emergency Medicine Journal, 20(1): 54-60. DOI: 10.1136/emj.20.1.54

Michel, P. 2003. Strengths and weaknesses of available methods for assessing the nature and scale of harm caused by the health system: Literature review. World Health Organization.

Moore, R. D., Smith, C. R., \& Lietman, P. S. 1984. Risk factors for the development of auditory toxicity in patients receiving aminoglycosides. Journal of Infectious Diseases, 149(1): 23-30.

Peloquin, C. A., Berning, S. E., Nitta, A. T., Simone, P. M., Goble, M., Huitt, G. A., \& Curran-Everett, D. 2004. Aminoglycoside toxicity: daily versus thrice-weekly 
dosing for treatment of mycobacterial diseases. Clinical Infectious Diseases, 38(11):1538-1544.

Pooran, A., Pieterson, E., Davids, M., Theron, G., \& Dheda, K. 2013. What is the cost of diagnosis and management of drug resistant tuberculosis in South Africa. Public Library of Science One, 8, e54587.

Schacht, J., Talaska, A. E., \& Rybak, L. P. 2012. Cisplatin and Aminoglycoside Antibiotics: Hearing Loss and Its Prevention. Anatomical Record (Hoboken), 295, 1837-1850. DOI:10.1002/ar.22578

Schellack, N., \& Naude, A. 2012. An overview of pharmacotherapy-induced ototoxicity. South African Family Practice, 55(4):357-366.

Selimoglu, E. (2007). Aminoglycoside-induced ototoxicity. Current Pharmaceutical Design. 13(1): 119-26.

Silman, S. \& Silverman, C. A. 1991. Auditory diagnosis: principles and applications. Academic Press.

Song, J. W., \& Chung, K. C. 2010. Observational studies: cohort and case-control studies. Plastic and Reconstructive Surgery, 126(6): 2234. DOI:10.1097/PRS.0b013e3181f44abc.

Suen, L. J., Huang, H. M., \& Lee, H. H. 2014. A comparison of convenience sampling and purposive sampling [Abstract]. Hu Li Za Zhi, 61(3): 105. DOI: 10.6224/JN.61.3.105

Theunissen, E. A., Dreschler, W. A., Latenstein, M. N., Rasch, C. R., van der Baan, S., de Boer, J. P., \& Zuur, C. L. 2014. A New Grading System for Ototoxicity in Adults. Annals of Otology, Rhinology \& Laryngology, 123(10):711-718.

Wang, S., Bian, Q., Liu, Z., Feng, Y., Lian, N., Chen, H., Hu, C., Dong, Y., \& Cai, Z. 1999. Capability of serum to convert streptomycin to cytotoxin in patients with aminoglycoside-induced hearing loss. Hearing Research, 137(1): 1-7.

Whitehorn, H., Sibanda, M., Lacerda, M., Spracklen, T., Ramma, L., Dalvie, S., \& Ramesar, R. 2014. High prevalence of cisplatin-induced ototoxicity in Cape Town, South Africa. SAMJ: South African Medical Journal, 104(4):288-291. 
World Health Organization. 2006. Guidelines for management of drug resistant tuberculosis. Geneva, (WHO/TB/2006.361).

World Health Organization. 2014. Global tuberculosis Report. Geneva, (WHO/HTM/TB/2014.08).

World Medical Association. 2013. World Medical Association Declaration of Helsinki: Ethical principles for medical research involving human subjects. Jama, 310(20): 2191.

Xie, J., Talaska, A. E., \& Schacht, J. 2011. New developments in aminoglycoside therapy and ototoxicity. Hearing Research, 281(1):28-37. DOI:10.1016/j.heares.2011.05.00 
Open Access available at: http://journals.uct.ac.za/index.php/UR DOI 10.15641/ur-at-uct.v1i2.38

\section{Appendix A: Abstraction form}

\begin{tabular}{|c|c|c|c|c|c|c|c|c|c|c|c|c|c|c|c|c|c|c|c|c|c|c|c|}
\hline \multirow{3}{*}{$\begin{array}{l}\mathrm{r} \\
\mathbf{5} \\
\mathrm{p} \\
\mathrm{n} \\
0 \\
\mathrm{e} \\
\mathrm{e} \\
\end{array}$} & \multirow{3}{*}{ Name or Folder \# } & \multirow{3}{*}{$\begin{array}{c}\text { DoB } \\
\text { dd-mm-ywy }\end{array}$} & \multirow{3}{*}{$\begin{array}{l}\text { Gender } \\
M / F\end{array}$} & \multirow{3}{*}{$\begin{array}{l}\text { Type and date of medical } \\
\text { intervention }\end{array}$} & \multirow{3}{*}{$\begin{array}{l}\text { Audiogram dare } \\
\text { Baseline } \\
\text { Firs Change } \\
\text { After inter. }\end{array}$} & \multirow{2}{*}{\multicolumn{2}{|c|}{ Tympanometry }} & \multicolumn{16}{|c|}{ Audiometry basel ine, interverention and sub sequent } \\
\hline & & & & & & & & \multicolumn{2}{|c|}{ sorte } & \multicolumn{2}{|c|}{ 5001t } & \multicolumn{2}{|c|}{ 10001te } & \multicolumn{2}{|c|}{ 2000+12 } & \multicolumn{2}{|c|}{ 30001 } & \multicolumn{2}{|c|}{ soutz } & \multicolumn{2}{|c|}{ 600018 } & \multicolumn{2}{|c|}{ sooutre } \\
\hline & & & & & & $t$ & R & Left & Right & Left & Righte & Left & right & Left & Righte & Left & Fight & Left & Right & Left & Fight & left & Right \\
\hline P1 & & & & & & & & & & & & & & & & & & & & & & & \\
\hline P2 & & & & & & & & & & & & & & & & & & & & & & & \\
\hline P3 & & & & & & & & & & & & & & & & & & & & & - & & \\
\hline P4 & & & & & & & & & & & & & & & & & & & & & & & \\
\hline $\mathrm{P5}$ & & & & & & & & & & & & & & & & & & & & & & & \\
\hline & & & & & & & & & & & & & & & & & & & & & & & \\
\hline P6 & & & & & & & & & & & & & & & & & & & & & & & \\
\hline D7 & & & & & & & & & & & & & & & & & & & & & & 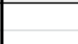 & \\
\hline & & & & & & & & & & & & & & & & & & & & & & & \\
\hline P8 & & & & & & & & & & & & & & & & & & & & & & & \\
\hline P9 & & & & & & & & & & & & & & & & & & & & - & & & \\
\hline P10 & & & & & & & & & & & & & & & & & & & & & & & \\
\hline & & & & & & & & & & & & & & & & 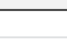 & & & & & & & \\
\hline P11 & & & & & & & & & & & & & & & & & & & & & & & \\
\hline
\end{tabular}

